\title{
A comparison of the relationship between depression, perceived disability, and physical performance in persons with chronic pain
}

\author{
Kevin N. Alschuler ${ }^{\mathrm{a}, \mathrm{b}}$, Mary E. Theisen-Goodvich ${ }^{\mathrm{a}}$, Andrew J. Haig ${ }^{\mathrm{a}}$, \\ Michael E. Geisser ${ }^{\mathrm{a}, *}$ \\ ${ }^{a}$ The Spine Program, Department of Physical Medicine and Rehabilitation, University of Michigan Health System, 325 E. Eisenhower Parkway, \\ Ann Arbor, MI 48108, United States \\ ${ }^{\mathrm{b}}$ Department of Psychology, Eastern Michigan University, Ypsilanti, MI, United States
}

Received 22 May 2007; received in revised form 24 October 2007; accepted 9 November 2007

Available online 21 December 2007

\begin{abstract}
This study examined the relationships between self-report of depressive symptoms, perceived disability, and physical performance among 267 persons with chronic pain. Prior research has reported a relationship between depression and disability using self-report measures. However, self-report instruments may be prone to biases associated with depression as depressed persons with pain may have an exaggerated negative view of their level of function. In addition, we examined whether the relationship between depression and functional activity was mediated by physiologic effort (as measured by heart rate). The results indicated that self-report of depressive symptoms (using the Center for Epidemiological Studies-Depression Scale (CES-D)) was significantly correlated with self-report of disability on the Quebec Back Pain Disability Scale (QBPDS) and physical performance on the Progressive Isoinertial Lifting Evaluation (PILE). Regression analyses revealed that depression assessed by the CES-D significantly contributed to the prediction of QBPDS scores and PILE performance even when controlling for age, gender, site of pain, and pain intensity. The magnitude of the relationships between depression and self-report and functional activity were similar, suggesting that a self-report bias associated with depression is not responsible for an observed relationship between depression and disability. Physiologic effort partially mediated the relationship between depression and physical performance. The findings further highlight the importance of depression in the experience of chronic pain.

(C) 2007 European Federation of Chapters of the International Association for the Study of Pain. Published by Elsevier Ltd. All rights reserved.
\end{abstract}

Keywords: Chronic pain; Chronic back pain; Depression; Disability

\section{Introduction}

Depression is common among persons with chronic pain. It is estimated that $30-54 \%$ of chronic pain patients suffer from severe forms of depression such as major depressive disorder (Banks and Kerns, 1996).

\footnotetext{
* Corresponding author. Tel.: +1 734615 1750; fax: +1 734615 1770 .

E-mail address: mgeisser@umich.edu (M.E. Geisser).
}

Given this high degree of co-morbidity, an important area of investigation involves the relationship between depression and function in persons with chronic pain. Persons with chronic pain and depression are less active and report greater disability and interference with daily activities due to pain compared to chronic pain patients without depression (Holzberg et al., 1996; Keogh et al., 2006). While the nature of this relationship has not been completely elucidated, depressive symptoms may contribute to a lack of motivation or effort which in turn 
results in less activity, and negative cognitions (e.g., hopelessness) may lead to pain beliefs that inhibit optimal function (Geisser et al., 2003).

Function and disability in chronic pain populations are frequently assessed using self-report measures. Measures of function such as ambulatory monitoring, performance on physical tasks, or observer ratings may be less prone to biases and are observable measures of behavior (Kop et al., 2005). Despite this, patients' perception of what activities they can or cannot engage in may be more robust predictors of daily activity levels. For example, Rudy et al. (2003) reported that perceived ability to perform a physical task and emotional functioning in a sample of persons with chronic pain were the best predictors of physical performance.

As depression and disability in pain populations are frequently examined using self-report measures, it is possible that a relationship between these two variables reflect a self-report bias that occurs in persons with mood disturbance (Smith et al., 1995; Hellstrom and Jansson, 2001). Persons with depression and chronic pain may make more negative assertions about his or her level of disability (Geisser et al., 2000b). For example, one study reported that depressed patients with chronic pain perceived themselves to be more disabled than non-depressed patients, even though nurse observers did not note this difference (Krause et al., 1994). Other research suggests that non-depressed persons also have a biased perception of their abilities, tending to overestimate their abilities or performance on a given task (Msetfi et al., 2005; Haaga and Beck, 2005). Both observations suggest that depression or absence of depression may bias self-report of function. Further research is needed to examine whether the relationship between depression and disability is due to a self-report bias.

Additionally, prior research in pain populations has not attended to factors such as submaximal effort that may mediate the relationship between depression and physical performance. As deficits in sustained attention and effortful cognitive tasks are prevalent in persons with major depression (Farrin et al., 2003; van der Meere et al., 2007; Zakzanis et al., 1998), subjects with depression and pain may demonstrate deficits in the ability to put forth maximal effort during physical activities.

The purpose of this investigation was to further examine the relationship between depression and disability in patients with chronic pain. Both self-report and observable measures of function were examined to determine whether the association between depression and function varied depending on how function was assessed. In addition, we wished to determine, whether the influence of depression on physical activity was mediated by physiologic effort during the task, as measured by heart rate. We predicted that depressive symptoms would be significantly related to both self-report of increased disability and poorer performance during assessment of physical performance, and that this relationship would be mediated by physiologic effort.

\section{Method}

\subsection{Subjects}

Subjects were 267 persons with chronic (three or more months) disabling pain who underwent a multidisciplinary assessment of their chronic pain at the University of Michigan Spine Program as part of their clinical care (Haig et al., 2006). All of the assessments were conducted on the same day. The evaluation consisted of functional testing, a physical therapy evaluation, psychological evaluation, evaluation by a rehabilitation counselor, and cardiovascular fitness testing. Persons in the sample had a mean age of 41.3 years $(\mathrm{SD}=$ 8.6), and a mean duration of pain of 57.8 months $(\mathrm{SD}=77.4)$. One hundred forty four persons $(53.9 \%)$ were male and $123(46.1 \%)$ were female. The large majority of patients had low back pain $(n=203)$, as defined by the International Association for the Study of Pain Primary Site of Pain Coding System (Bonica, $1990)$. Neck pain $(n=31)$, thoracic pain $(n=15)$ and pain in three or more body sites $(n=11)$ were the next three most common sites of pain. Most patients reported their amount of education to be some college $(n=104)$, while 73 reported being high school graduates, 42 completed some high school, 25 were college graduates, 23 completed graduate or professional school, and one person provided missing data. The sample was predominantly Caucasian $(n=246)$, but 19 persons were African American, and two were Hispanic.

\subsection{Measures}

\subsubsection{Demographic questionnaire}

Individuals completed a questionnaire that solicits information regarding duration of pain, age, education, race, and other demographic and pain information. For the correlational or regression analyses, categorical variables were dummy coded. For gender, males were coded as 0 , and females as 1 .

\subsubsection{Pain}

All subjects completed the McGill Pain Questionnaire (MPQ; (Melzack, 1975)). The MPQ measures subjective pain experience quantitatively, and consists of twenty groups of single word pain descriptors with the words in each group increasing in rank order intensity. The sum of the rank values for each descriptor based on its position in the word set results in a score termed the Pain Rating Index (PRI). There are also three major 
subscales of the MPQ which assess the sensory, affective and evaluative dimensions of pain experience. The total PRI was used in the present study as the measure of selfreport of pain intensity. Repeat administration of the MPQ has revealed a $70.3 \%$ rate of consistency in the PRI score (Melzack, 1975).

\subsubsection{Depression self-report}

Self-report of depressive symptoms was assessed using the Center for Epidemiological Studies Depression Scale (CES-D; (Radloff, 1977)). The CES-D is a 20-item scale of depressive symptoms where persons are asked to rate the frequency of symptoms on a $0-3$ scale in relation to how they felt during the past week. A total score is obtained by summing the responses to all of the items, and higher scores reflect greater depressive symptoms. The validity of the scale is reported to not be compromised in medical populations, and studies suggest that the CES-D has good concurrent validity with depression diagnoses established through clinical interview in chronic pain populations (Berkman et al., 1986; Geisser et al., 1996; Turk and Okifuji, 1994).

\subsubsection{Disability self-report}

Self-report of disability was measured using the Quebec Back Pain Disability Scale (QBPDS; (Kopec et al., 1995). The QBPDS is a 20 -item scale on which patients are asked to rate the amount of difficulty they have performing activities of daily living, such as getting out of bed, walking several miles, and making a bed. Patients are asked to rate their degree of difficulty ranging from zero "not difficult at all" to five "unable to do". Total scores, computed by summing the responses to each item, range from $0-100$. Validation of the questionnaire revealed test-retest reliability to be 0.93 and internal consistency to be 0.95 (Kopec et al., 1995).

\subsubsection{Physical performance}

Physical performance was based on the Progressive Isoinertial Lifting Evaluation (PILE; (Curtis et al., 1994; Mayer et al., 1988a,b). The PILE entails a floor to waist $(\mathrm{F}-\mathrm{W})$ lift $(30 \mathrm{in}$.$) , and a waist to shoulder$ (W-S) lift (30-54 in.). Men start at $10 \mathrm{lb}$, and women start at $5 \mathrm{lb}$, and are asked to perform four repetitions of the lift at each weight. Following the completion of each lift, they are asked if they believe they can lift more weight, or if they wish to stop the task. If they indicate they can lift more weight, the total weight is increased by $5 \mathrm{lb}$. Those who progressively lift more weight are stopped when they reach their maximum predicted weight ( $60 \%$ of ideal body weight) based on their gender and height. Ideal body weight was calculated from the tables presented by Mayer et al. (1988a,b). Lifting performance was expressed as the ratio of actual to expected weight lifted to control for anthropometric differences between subjects (Haig et al., 2006; Mailloux et al., 2006). Lower scores (percentages) on this measure are associated with poorer physical performance.

Performance on the PILE has been shown to change significantly over time among persons undergoing treatment for back pain (Mayer et al., 1988a,b; Curtis et al., 1994), and is sensitive to changes in clinical status among persons with chronic spinal pain (Ljungquist et al., 2006). The PILE has also been found to have high test-retest reliability (Lygren et al., 2005; Smeets et al., 2006), when assessed using intraclass correlation coefficients, however Smeets et al. (2006) have questioned, whether measurement error in the PILE is too high for the test to be of clinical value.

\subsubsection{Heart rate}

Heart rate (HR) was recorded during the PILE using a transmitter strapped across the chest, and a wireless monitor (Pulse model, Polar Electro, Inc., Woodbury, NJ). Percent of maximum heart rate during the PILE was calculated by dividing the patients' maximum heart rate by their maximum heart rate based on age (220 beats/minute - age; (American College of Sports Medicine, 1986)). While one purpose of measuring heart rate during the PILE is for subject safety, Mayer et al. $(1988 \mathrm{a}, \mathrm{b})$ suggest that heart rate on the PILE can also reflect subject effort among persons not taking heart rate limiting medications. Haig et al. (2006) suggest that heart rate on tests such as the PILE and cycle ergometer testing is a measure of physiologic effort. Prior research has also demonstrated that heart rate increases are highly associated with greater perceived exertion and other physiological parameters during strenuous physical activity (Borg, 1973). In the present sample, percent maximum HR was significantly correlated with performance on the PILE F-W $(r=0.51, p<0.001)$ and the PILE W-S $(r=0.47, p<0.001)$.

\subsection{Analyses}

Pearson correlation coefficients were calculated to determine relationships between disability, depression, demographic variables, and pain intensity. To examine the similarity between the correlations, statistical tests were performed to examine whether the correlations between depression and the two function measures were statistically significant from each other. A $t$-test was used for comparing two correlation coefficients from related samples (Weinberg and Goldberg, 1979). Three simultaneous multiple regressions were conducted to examine the influence of self-report of depressive symptoms on self-report of disability and physical performance while controlling for age, gender, site of back pain, and pain intensity. Thus, all variables were entered together, and the beta weights in these analyses reflect the independent contribution of each variable. Site of pain was dummy coded using four variables to denote 
five pain locations (low back pain, neck pain, thoracic pain, pain in three or more sites, and other).

Finally, mediation analyses, as proposed by Baron and Kenny (1986), were conducted to assess whether physiologic effort (as measured by HR) mediated the relationship between depression and physical performance on the PILE. According to the authors, mediation exists when: (1) an independent variable (in this case, depression) significantly predicts a dependent measure (function); (2) the independent variable is significantly associated with the mediator variable (HR); and (3) the mediator significantly predicts the dependent variable when controlling for the independent variable. If these conditions are met and the association between the independent and dependent variable becomes 0 when controlling for the mediator, complete mediation is said to occur. When this association is not eliminated, but significantly reduced, this reflects partial mediation. A Sobel test was used to examine whether the total indirect effect of depression on functional performance as mediated by heart rate was statistically significant. A macro provided by Preacher and Hayes (2004) was used in SPSS to calculate the statistics for the Sobel test. To control for age, gender, site of pain, and pain intensity, residual values of self-report of depression, physical performance on the PILE, and HR were calculated using multiple regression, and these values were used to compute the Sobel test statistics.

\section{Results}

Means and standard deviations for the various measures used in the analyses are presented in Table 1.

Table 1

Sample means and standard deviations on study measures

\begin{tabular}{ll}
\hline Variable & Mean (SD) \\
\hline MPQ & $31.7(11.8)$ \\
CES-D & $24.5(13.2)$ \\
Quebec & $58.5(18.2)$ \\
PILE F-W & $0.38(0.24)$ \\
PILE W-S & $0.45(0.27)$ \\
\hline
\end{tabular}

Pearson correlation coefficients calculated between the measures of disability, self-report of depression, demographic variables, and pain intensity are presented in Table 2. The measures of disability were significantly correlated with each other (self-report and PILE F-W; $r=-0.47, \quad p<0.001 ;$ self-report and PILE W-S; $r=-0.39, p<0.001 ;$ PILE F-W and PILE W-S; $r=0.72, p<0.001$ ). Pain intensity was significantly correlated with self-report of depression $(r=0.19, p<0.01)$ and self-report of disability $(r=0.18, p<0.01)$, but not with performance on either of the PILE measures.

With regard to the associations between depression and the measures of function, number of self-report depressive symptoms were significantly associated with greater disability on the Quebec $(r=0.31, p<0.001)$ and poorer performance on the PILE F-W and PILE $\mathrm{W}-\mathrm{S}(r=-0.25, p<0.001$ and $r=-0.24, p<0.001$, respectively). Using the aforementioned Weinberg and Goldberg (1979) statistic to assess differences between correlations, and ignoring the sign of correlation coefficient due to scaling differences (i.e., greater disability on the QBPDS is reflected in a higher score, while greater disability on the PILE is reflected by a lower score PILE), the correlation between self-report of depression and scores on the QBPDS was not significantly different from the correlation between CES-D scores and performance on the PILE F-W test $(t=1.00, p=0.16)$. Similarly, the correlation between CES-D and QBPDS scores did not significantly differ from the correlation between CES-D scores and performance on the PILE $\mathrm{W}-\mathrm{S}(t=1.09, p=0.14)$.

Three simultaneous multiple regressions were conducted to examine the influence of self-report of depressive symptoms on self-report of disability, PILE F-W, and PILE W-S while controlling for age, gender, site of pain, and pain intensity. The beta weights (standardized regression coefficients) and the associated levels of significance for all the regressions are reported in Table 3. The first regression explored the relative contribution of self-report of depression to self-report of disability. The multiple regression coefficient with all variables entered was $0.34(F=4.34, p<0.001)$, and all the variables combined accounted for $12 \%$ of the variance in

Table 2

Correlations between sex, age, pain (MPQ), self-report of depression (CES-D), self-report of disability (Quebec), and functional activity (PILE floor to waist lift and waist to shoulder lift)

\begin{tabular}{lllllll}
\hline Variable & 2 & 3 & 4 & 5 & 6 & 7 \\
\hline 1. Sex & -0.10 & -0.10 & 0.02 & 0.02 & 0.07 & 0.02 \\
2. Age & - & 0.00 & $-0.13^{*}$ & -0.04 & -0.08 \\
3. MPQ & - & - & $0.19^{* *}$ & $0.18^{* *}$ & $-0.25^{* * *}$ \\
4. CES-D & - & - & - & $-0.31^{* * *}$ & -0.09 \\
5. Quebec & - & - & - & - & $-0.47^{* * *}$ \\
6. PILE F-W & - & - & - & - & $-0.39^{* * *}$ \\
7. PILE W-S & - & & & - & $0.72^{* * *}$ \\
\hline
\end{tabular}

Note: ${ }^{*} p<0.05,{ }^{* *} p<0.01,{ }^{* * *} p<0.001$. 
Table 3

Beta weights from regression analyses of the influence of age, gender, pain intensity and depression on function

\begin{tabular}{llll}
\hline Independent variable & Disability measure & \\
\cline { 2 - 3 } & PILE F-W & PILE W-S \\
\hline Age & 0.00 & -0.01 & 0.01 \\
Sex & 0.03 & - & 0.07 \\
Pain site & - & -0.16 & - \\
Neck pain & -0.06 & -0.11 & -0.03 \\
Thoracic pain & -0.14 & -0.31 & -0.04 \\
Low back pain & -0.10 & -0.12 & -0.10 \\
Three or more sites & -0.03 & -0.02 & -0.02 \\
MPQ & $0.13^{*}$ & $-0.25^{* *}$ & 0.01 \\
CES-D & $0.28^{* *}$ & $-0.23^{* * *}$ \\
\hline
\end{tabular}

Notes: Simultaneous regressions were conducted separately for the Quebec and the PILE F-W and PILE W-S. ${ }^{*} p<0.05,{ }^{* *} p<0.01,{ }^{* * *} p<0.001$.

self-report of disability. Higher levels of self-report of depression $(t=4.76, p<0.001)$ and higher pain levels $(t=2.12, p<0.05)$ significantly predicted higher levels of self-report of disability. No other variables independently contributed to the prediction of self-report of disability.

The second regression explored the relative contribution of self-report of depression to physical performance measured during the PILE F-W test. The multiple regression coefficient with all variables entered was 0.30 $(F=3.13, p<0.01)$, accounting for $9 \%$ of the variance in physical performance. Higher levels of self-report of depression $(t=-4.01, p<0.001)$ significantly predicted lower levels of performance on the PILE F-W. No other variables contributed independently to the prediction of physical performance on the PILE F-W.

The third regression examined the relative contribution of self-report of depression to physical performance during the PILE $\mathrm{W}-\mathrm{S}$. The multiple regression coefficient with all variables entered was $0.33(F=3.82, p<0.01)$, and the variables combined accounted for $11 \%$ of the variance in this measure. Higher levels of self-report of depression $(t=-3.84, p<0.001)$ significantly predicted lower levels of physical performance on the waist to shoulder lift, and gender also significantly predicted performance $(t=-3.46, p<0.01)$. Women tended to perform more poorly compared to men.

Finally, we explored whether the relationships between depression and physical performance observed were mediated by physiologic effort (as measured by HR). Two of the subjects were missing HR data, so the sample size for this analysis was 267 . The relationships are shown in Fig. 1 separately for the PILE floor-to-waist lift, and the waist-to-shoulder lift. Age, gender, site of pain, and pain intensity were controlled for in the computation of all path coefficients. Results indicate that, while the direct relationship between depression and PILE F-W remains significant, the value of the path coefficient is reduced by approximately half in comparison to the initial regression analysis. In addition, the indirect pathway whereby physiologic effort

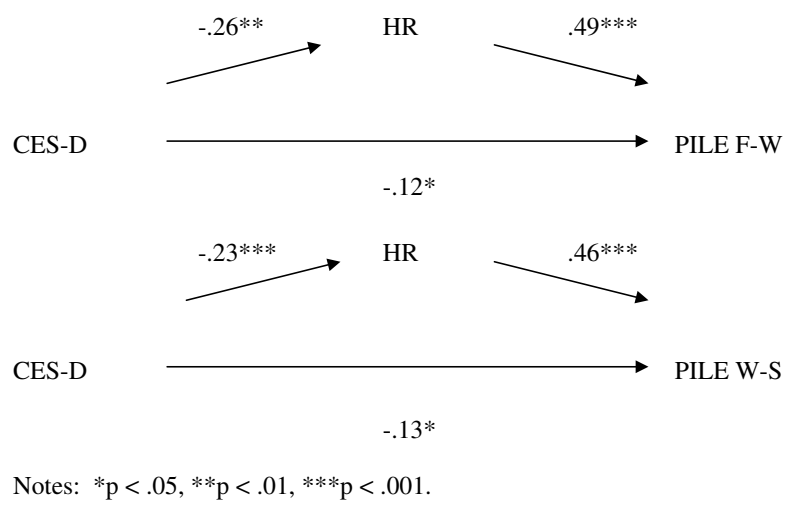

Fig. 1. Heart rate as a mediator of the relationship between depression and physical performance on the PILE controlling for age, gender, site of back pain, and pain intensity.

(HR) mediates that relationship between depression and physical performance is also significant. That is, the path between depression and physiologic effort is significant, and the path coefficient between physiologic effort and physical performance measured during the PILE F-W is also statistically significant. The Sobel test for the total indirect effect of depression on PILE F-W through $\mathrm{HR}$ was also statistically significant $(Z=$ $-3.94, p<0.001)$. For the PILE W-S, the findings were almost identical. Again, the Sobel test for the total indirect effect of depression on PILE W-S through HR supported the hypothesis that HR acts as a mediator $(Z=-3.47, p<0.001)$.

\section{Discussion}

The current study explored the association between self-report of depressive symptoms, self-report of disability, and objectively measured physical performance. The goal was to ascertain whether a significant relationship between depression and disability could be obtained, and if this relationship could be explained by a self-report bias. Correlation analyses revealed 
significant relationships between depression and the two disability measures examined. Regression analyses indicated that self-report of depression significantly contributed to higher levels of self-report of disability and physical performance, even when controlling for age, gender, site of pain, and pain intensity. Together, these analyses support the notion that there is a genuine relationship between depression and disability in persons with chronic back pain, regardless of the measurement technique utilized for disability.

These results support the findings of previous studies indicating that depression significantly influences disability associated with chronic pain (Geisser et al., 2000a; Holzberg et al., 1996; Keogh et al., 2006). Additionally, it has been reported that in comparison to non-depressed patients, chronic pain patients with depression perceive themselves as more disabled (Krause et al., 1994). Using the guidelines proposed by Cohen (1988) for small $(r=0.10)$, moderate $(r=0.30)$ and large $(r=0.50)$ effect sizes for behavioral science research, the relationships observed between depression and the three measures of physical performance $(r ' s=0.24-0.31)$ examined in the present study either approached or just exceeded a moderate effect size. However, depression in these instances only accounted for $5.8-9.6 \%$ of the variance in disability, bringing into question the clinical significance that altering depression might have on function in persons with chronic pain. In addition, many factors have been proposed to be related to disability associated with chronic pain (Holzberg et al., 1996; Rudy et al., 2003), and altering depression or any other factor in isolation may not be sufficient to significantly improve function. Further research is needed to determine the impact of targeted treatment for depression on functional outcomes in persons with chronic pain.

The mediation analyses indicated that physiologic effort, as measured by maximum HR during the PILE $\mathrm{F}-\mathrm{W}$ and PILE W-S, partially mediated the relationship between depression and physical performance on the PILE. The total indirect effect of depression on physical performance through its association with HR was statistically significant, and the direct effect on depression on PILE performance was also statistically significant, although much reduced compared to the zero-order correlation. These findings support the notion that depression influences function at least in part through reduced physiologic effort during activity (Geisser et al., 1994). It is possible that neurovegetative symptoms associated with depression, such as anergia and anhedonia, limit a person's capacity to put forth effort on various tasks and/or decrease their interest or motivation in engaging in effortful activity. It also plausible that negative cognitions also reduce effort during activity, as persons with depression may have more negative views about the outcome of their efforts, or be more fearful that activity may cause more pain and discomfort. Future studies examining how the various dimensions of depression impact effort and function would be useful.

The present study suggests that the depression-disability relationship extends beyond self-report measures, as it is maintained when objective measures of disability are employed. Thus, it appears that potential self-report biases reported to be associated with depression do not explain the observed relationship between depression and disability among persons with chronic pain. This is important in the context of research on self-report biases, as the present results differ from a previous study that found depressed patients perceived themselves as more disabled than objective observers rated them to be (Krause et al., 1994). However, these results are consistent with a previous study among persons with rheumatoid arthritis (Smith et al., 1995). The findings have implications regarding the impact of depression on the assessment of different experiences associated with chronic pain, particularly given the high prevalence of depression reported in this population. The findings of the present study suggest that self-report measures are not confounded by such biases.

In addition to the findings regarding self-report biases, it should be noted that pain levels significantly contributed to the prediction of self-report of disability, but not objectively reported disability. It is possible that this relationship may be explained in part by the instructions on measures such as the QBPDS, as the questionnaire asks participants to relate their disability to their pain, thus implying a link between pain and disability (Kopec et al., 1995). The absence of a significant relationship between pain intensity and physical performance is consistent with previous research assessing the relationship of pain to disability (Millard et al., 1991; Geisser et al., 2000a). Research on fear-avoidance models of pain have suggested that fear of pain rather than actual pain experienced during a functional task is a more potent predictor of function (Waddell et al., 1993; Crombez et al., 1999). In addition, research suggests that persons are highly variable in terms of how they respond to pain, as pain may have a limited impact on the function of some persons, and a significant impact on disability in others (Millard et al., 1991). Finally, these results add to literature suggesting that the subjectivity of pain makes it a poor predictor of disability (Robinson, 2001).

The design of the present study has both strengths and limitations. This study benefited from having a large sample size and of a mixture of pain problems. However, it is recognized that the majority of the sample was comprised of persons with back pain problems, particularly low back pain, limiting the generalizability of the findings to all chronic pain populations. In addition, the analyses consisted of testing the hypotheses on crosssectional data, thus causal inferences about relationships 
in the data cannot be made. Future studies would benefit from employing experimental or longitudinal designs that would allow one to examine cause-effect relationships between depression and disability. In addition, heart rate on the PILE has not been well validated as a measure of physiologic effort, and can be influenced by multiple factors such as fitness and medications. For example, antidepressant medications can influence heart rate activity, as one possible side-effect of these medications, in addition to electrocardiogram changes and arrhythmias, is tachycardia. However, use of antidepressant medications among persons with depression cannot account for the observed relationships in the study, as one would expect tachycardia produced by these medications to produce a positive relationship between depression and heart rate, not a negative relationship as was observed in the data. Finally, factors other than physiologic effort are associated with this variable during effortful activity.

It should also be noted that the reliability and clinical utility of the PILE has been questioned in a recent study by Smeets et al. (2006). These authors, based on an observed value of a limits of agreement statistic, suggest that the measurement variability of the PILE is too high, and concluded that the test is not clinically useful. However, pain measures may not be highly stable over time as pain itself constantly fluctuates, and because of this, measures of test-retest reliability have limited utility as estimates of the reliability of pain measures (Jensen, 2003). Thus, although the PILE may not be stable over time as reported by Smeets et al. (2006), we would suggest that the clinical utility of the PILE needs to also be examined in the context of its sensitivity to changes in pain. One study reported that the PILE is highly sensitive to change in clinical status among persons with chronic spinal pain (Ljungquist et al., 2006).

The findings of the present study also raise questions regarding the need for specific treatment of depression in persons with chronic pain conditions. It would be beneficial to examine whether pharmacological or cognitivebehavioral therapies that specifically target depression symptoms among persons with chronic pain and depression improve treatment outcomes such as function and pain compared to interventions that, for example, solely target pain.

\section{References}

American College of Sports Medicine. Guidelines for exercise testing and prescription. 3rd ed. Philadelphia, PA: Lea\&Febiger; 1986.

Banks SM, Kerns RD. Explaining high rate of depression in chronic pain: a diathesis-stress framework. Psychol Bull 1996;119:95-110.

Baron RM, Kenny DA. The moderator-mediator variable distinctions in social psychological research: conceptual, strategic, and statistical considerations. J Pers Soc Psychol 1986;51:1173-82.
Berkman LF, Berkman CS, Kasl S. Depressive symptoms in relation to physical health and functioning in the elderly. Am J Epidemiol 1986;124:372-88.

Bonica JJ. Definitions and taxonomy of pain. 2nd ed. In: Bonica JJ, editor. The management of pain, vol. 1. Philadelphia, PA: Lea and Febiger; 1990. p. 18-27.

Borg GA. Perceived exertion: a note on "history" and methods. Med Sci Sports 1973;5:90-3.

Cohen J. Statistical power analysis for the behavioral sciences. 2nd ed. Hillsdale, NJ: Lawrence Erlbaum Associates; 1988.

Crombez G, Vlaeyen JWS, Heuts PHTG, Lysens R. Pain-related fear is more disabling than pain itself: evidence on the role of painrelated fear in chronic back pain disability. Pain 1999;80:329-39.

Curtis L, Mayer TG, Gatchel RJ. Physical progress and residual impairment quantification after functional restoration. Part III: isokinetic and isoinertial lifting capacity. Spine 1994;19:401-5.

Farrin L, Hull L, Unwin C, Wykes T, David A. Effects of depressed mood on objective and subjective measures of attention. J Neuropsychiatr Clin Neurosci 2003;15:98-104.

Haig AJ, Geisser M, Michel B, Theisen M, Yamakawa K, Buccholz R, et al. The spine team assessment I: a codified multidisciplinary assessment for persons with chronic back pain disability. Disabil Rehabil 2006;28:1071-8.

Hellstrom C, Jansson B. Psychological distress and adaptation to chronic pain: symptomology in dysfunctional, interpersonally distressed, and adaptive copers. J Musculoskelet Pain 2001;9:51-67.

Geisser ME, Robinson ME, Keefe FJ, Weiner ML. Catastrophizing, depression and the sensory, affective and evaluative aspects of chronic pain. Pain 1994;59:79-83.

Geisser ME, Roth RS, Bachman JE, Eckert TA. The relationship between symptoms of post-traumatic stress disorder and affective disturbance, pain and disability among patients with accident and non-accident related pain. Pain 1996;66:207-14.

Geisser ME, Haig AJ, Theisen ME. Activity avoidance and function in persons with chronic back pain. J Occup Rehabil 2000a;10:215-27.

Geisser ME, Roth RS, Theisen ME, Robinson ME, Riley J. Negative affect, self-report of depressive symptoms, and clinical depression: relation to the experience of chronic pain. Clin $\mathrm{J}$ Pain 2000b; $16: 110-20$.

Geisser ME, Robinson ME, Miller QL, Bade SM. Psychosocial factors and functional capacity evaluation among persons with chronic pain. J Occup Rehabil 2003;13:259-76.

Haaga DAF, Beck AT. Perspective on depressive realism: implications for cognitive theory of depression. Behav Res Therap 2005;33:41-8.

Holzberg A, Robinson ME, Geisser ME, Gremillion HA. The effects of depression and chronic pain on psychosocial and physical functioning. Clin J Pain 1996;12:118-25.

Jensen MP. Questionnaire validation: a brief guide for readers of the research literature. Clin J Pain 2003;19:345-52.

Keogh E, McCracken LM, Eccleston C. Gender moderates the association between depression and disability in chronic pain patients. Eur J Pain 2006;10:413-22.

Kop WJ, Lyden A, Berlin AA, Ambrose K, Olsen C, Gracely RH, et al. Ambulatory monitoring of physical activity and symptoms in fibromyalgia and chronic fatigue syndrome. Arthritis Rheum 2005;52:296-303.

Kopec JA, Esdaile JM, Abrahamowicz M, Abenhaim L, WoodDauphinee S, Lamping DL, et al. The Quebec Back Pain Disability Scale measurement properties. Spine 1995;20:341-52.

Krause SJ, Wiener RL, Tait RC. Depression and pain behavior in patients with chronic pain. Clin J Pain 1994;10:122-7.

Ljungquist T, Nygren A, Jensen I, Harms-Ringdahl K. Physical performance tests for people with spinal pain-sensitivity to change. Disabil Rehabil 2006;25:856-66.

Lygren H, Dragesund T, Joensen J, Ask T, Moe-Nilssen R. Test-retest reliability of the Progressive Isoinertial Lifting Evaluation (PILE). Spine 2005;30:1070-4. 
Mailloux J, Finno M, Rainville J. Long-term exercise adherence in the elderly with chronic low back pain. Am J Phys Med Rehabil 2006;85:120-6.

Mayer T, Barnes D, Kishino N. Progressive isoinertial lifting evaluation I. A standardized protocol and normative data base. Spine 1988a;13:993-7.

Mayer T, Barnes D, Nichols G. Progressive isoinertial lifting evaluation II. A comparison with isokinetic lifting in a disabled chronic low-back pain industrial population. Spine 1988b;13:998-1002.

Melzack R. The McGill pain questionnaire: major properties and scoring methods. Pain 1975;1:277-99.

Millard RW, Wells N, Thebarge RW. A comparison of models describing reports of disability associated with chronic pain. Clin $\mathbf{J}$ Pain 1991;7:283-91.

Msetfi RM, Murphy RA, Simpson J, Kornbrot DE. Depressive realism and outcome density bias in contingency judgments: the effect of the context and intertrial interval. Exp Psychol 2005;134:10-22.

Preacher KJ, Hayes AF. SPSS and SAS procedures for estimating indirect effects in simple mediation models. Behav Res Meth Instru Comput 2004;36:717-31.

Radloff L. The CES-D scale: a self-report depression scale for research in the general population. Appl Psychol Meas 1977;1:385-401.
Robinson JP. Disability evaluation in painful conditions. In: Turk DC, Melzack R, editors. Handbook of pain assessment. 2nd ed. New York, NY: Guilford Press; 2001. p. 248-72.

Rudy TE, Lieber SJ, Boston JR, Gourley LM, Baysal E. Psychosocial predictors of physical performance in disabled individuals with chronic pain. Clin J Pain 2003;19:18-30.

Smeets RJ, Hijdra HJ, Kester AD, Hitters MW, Knottnerus JA. The usability of six physical performance tasks in a rehabilitation population with chronic low back pain. Clin Rehabil 2006;20:989-97.

Smith CA, Wallston KA, Dwyer KA. On babies and bathwater: disease impact and negative affectivity in the self-reports of persons with rheumatoid arthritis. Health Psychol 1995;14:64-73.

Turk DC, Okifuji A. Detecting depression in chronic pain patients: adequacy of self-reports. Behav Res Therap 1994;32:9-16.

van der Meere J, Borger N, van OsT. Sustained attention in major unipolar depression. Percept Mot Skills 2007;104:1350-4.

Weinberg SL, Goldberg KP. Basic statistics for education and the behavioral sciences. Boston, MA: Houghton Mifflin Company; 1979, p. 412.

Waddell G, Newton M, Henderson I, Somerville D, Main CJ. A fearavoidance beliefs questionnaire (FABQ) the role of fear-avoidance beliefs in chronic low back pain and disability. Pain 1993;52:157-68.

Zakzanis KK, Leach L, Kaplan E. On the nature and pattern of neurocognitive function in major depressive disorder. Neuropsychiatr Neuropsychol Behav Neurol 1998;11:111-9. 